\title{
Test suite minimization for testing in context
}

\author{
Nina Yevtushenko \\ Tomsk State University \\ 36, Lenin str., Tomsk, 634050, Russia \\ Yevtushenko.RFF@elefot.tsu.tomsk.su \\ Ana Cavalli, Luiz Lima Jr. ${ }^{1}$ \\ National Institute of Telecommunications \\ 9, rue Charles Fourier, 91011 Evry Cedex, France \\ \{Ana.Cavalli,lima\}@hugo.int-evry.fr
}

\begin{abstract}
In this paper, the problem of test suite minimization for testing in context is studied and some results are proposed. The test architecture considered is general enough to allow the context and the component to have external inputs and outputs. Using a given fault model for testing in context, conditions are provided to detect all the redundant transitions that do not need to be tested. A complete test suite for the fault model is obtained and a method to select a minimal subset of this test suite with the same fault detection power is then proposed. Furthermore, the proposed method can be used to reduce a test suite produced by human experts while preserving its fault detection power.
\end{abstract}

\section{Keywords}

Test derivation, test in context, embedded testing, test of communicating FSMs.

\section{INTRODUCTION}

As the complexity of communication protocols increases, there is a strong need for systematic methods for test derivation with guaranteed fault coverage based on formal description techniques. Formal description techniques for communication protocols usually use a system of communicating finite state machines (FSMs) as their

1. Work funded by CNPq. 
underlying model. The 'black-box' testing of a system can be performed by constructing the composite machine that describes a behaviour of a system at points that are accessible for testing and then applying test suite derivation methods for FSMs [Vasilevsky 73][Chow 78][Vuong 89][Fujiwara 91][Petrenko 96-1][Yannakakis 95]. However, this approach provides very long tests due to a huge number of states of the composite machine.

If only some components of the system need testing, the problem of testing an embedded component machine (or 'gray-box' testing [IS-9646 91] or testing in context [Petrenko 96-2]) appears. In this case, a specification system is represented as a composition of two FSMs. One of these machines, called component, is the composite FSM of all machines which need testing. The other machine, called context, is the composite machine of all other machines that are assumed to be faultyfree. If the set of Points of Control and Observation (PCOs) of a system contains all the access points to the component (i.e. there are no internal signals exchanged between the component and the context), then testing in context reduces to 'blackbox' testing in isolation and a number of methods can be called for deriving a test suite with guaranteed fault coverage. Otherwise, 'black-box' testing at the PCOs will (unnecessarily) test the context as well, that is assumed to be faulty-free. The issue has been studied in a number of papers [Lima 97][Petrenko 97][Petrenko 962][Huang 96][Lee 96][Kim 95][PT 96]. Nevertheless, as far as we know, the application of embedded testing methods in precise fields has not been greatly explored [Lima 98].

Not all transitions of the system come from the combination of transitions of the context and the component. Some concern uniquely the context and thus, do not need testing. Various methods for selecting such transitions have been proposed. Based on the so-called fault function, the authors of paper [Petrenko 96-2] suggest a method for translation of component's transfer and output faults into faults of the composite FSM of the specification and call a method elaborated in [Petrenko 92] for complete test suite derivation. Another approach is based on a coverage of component's transitions by performing a random walk of the specification composite FSM [Lee 96] but fault detection power of the method is unknown. Paper [Lima 97] is devoted to determining a certain part of the composite machine comprising transitions should be tested in the case when any fault of the component does not increase the number of states of the specification system. In the paper, transitions which the component does not affect are called redundant transitions while other transitions are called suspicious transitions. If the context is faulty-free then an external sequence traversing only redundant transitions is superfluous and can be deleted from the test suite without loss of its completeness. A method for determining such transitions is proposed, and sufficient conditions for deleting superfluous test cases are established. The authors notice, nevertheless, that a more rigorous analysis is necessary to elaborate a method for checking suspicious transitions. This paper continues this work.

The paper is structured as follows. After the preliminaries of Section 2, Section 3 explains how to deal with transitions concerning only the faultless context 
(redundant transitions) in the machine representing the system's global behaviour. In Section 4, given a set of external input sequences (from Section 3), we propose a method for derivation of a regular language describing a part of the component's behaviour that can be tested with the set and a complete test suite minimization technique when the obtained regular set is finite. We conclude with a discussion on the future work in Section 5.

\section{PRELIMINARIES}

\subsection{Finite State Machines}

A Finite State Machine (FSM) (often simply called 'a machine' throughout this paper) is an initialized completely specified deterministic Mealy machine which can be formally defined as follows. A FSM $A$ is a 6-tuple $\left(S, X, Y, \delta, \lambda, s_{0}\right)$ where $S$ is a finite set of states with $s_{0}$ as the initial state; $X$ - a finite nonempty set of input symbols; $Y$ - a finite nonempty set of output symbols, $\delta$ and $\lambda$ are the next state and output functions $\delta: S \times X \rightarrow S$ and $\lambda: S \times X \rightarrow Y$. In usual way, functions $\delta$ and $\lambda$ are extended to functions on the set $S \times X^{*}$ where $X^{*}$ is the set of all finite input sequences including the empty sequence $\varepsilon$. The FSM $A$ is said to be connected if each state of $A$ is reachable from the initial state, i.e. for each state $s \in S$ there exists an input sequence $\alpha \in X^{*}$ such that $\delta\left(s_{0}, \alpha\right)=s$.

Two states $s_{i}$ and $s_{j}$ are said to be distinguishable states of $A$ if there exists an input sequence $\alpha \in X^{*}$ such that the FSM $A$ produces different output sequences at the states $s_{i}$ and $s_{j}$ to the $\alpha$; otherwise, states $s_{i}$ and $s_{j}$ are said to be equivalent. An FSM with pairwise distinguishable states is called a minimal FSM.

Given sequences $\alpha=x_{1} \ldots x_{k} \in X^{*}$ and $\beta=y_{1} \ldots y_{k} \in Y^{*}$, the sequence $x_{1} y_{1} \ldots x_{k} y_{k}$ is called a trace of the FSM $A$ if $\beta=\lambda\left(s_{0}, \alpha\right)$. For a sequence $\sigma$ over alphabets $X$ and $Y$, $X \cap Y=\varnothing$, the $X$-projection of $\sigma$ is obtained by deleting from $\sigma$ all symbols of the set $Y$.

Given an FSM $B=\left(T, X, Y, \psi, \varphi, t_{0}\right), B$ is said to be equivalent to $A$, written $B \cong A$, if for any sequence $\alpha \in X^{*}$, it holds that $\lambda\left(s_{0}, \alpha\right)=\varphi\left(t_{0}, \alpha\right)$, otherwise $B$ is distinguishable from $A$, written $B \neq A$. In other words, $B$ is equivalent to $A$ if $A$ and $B$ exhibit the same behaviour under all input sequences. Protocol conformance testing is often formalized as FSMs equivalence problem.

\subsection{Fault models}

A fault model is a triple $<A, \sim, \Re>$ [Petrenko 96-2], where $A$ is a reference FSM, $\Re$, usually called a fault domain, is the set of all (possibly faulty) implementation FSMs defined over the same input alphabet as the reference machine, and $\sim$ is a conformance relation. If the reference FSM $A$ is a deterministic minimal FSM with $n$ states, $\Re_{n}$ is a set of all deterministic FSMs over the same input alphabet as $A$ with at most $n$ states and $\sim$ is the equivalence relation $\cong$ then the fault model $\angle A, \cong, \Re_{n}>$ is a classical 'black-box' model. A complete test suite $T S$ w.r.t. the fault model $\angle A$, $\Re_{n}>$ is a finite set of finite input sequences of the reference FSM $A$ such that for any 
FSM $B \in \Re_{n}$, if $B \neq A$ then there exists input sequence $\alpha \in T S$ such that $A$ and $B$ produce different output sequences to $\alpha$. There exists a number of methods for complete test suite derivation w.r.t. this model. Below we sketch the Wp-method [Fujiwara 91] for a complete test derivation.

Let $A=\left(S, X, Y, \delta, \lambda, s_{0}\right)$ be a minimal connected FSM with $n$ states. A subset $V \subseteq X^{*}$ comprising the empty sequence $\varepsilon$ is called a state cover set if for each state $s \in S$, there exists a sequence $\alpha \in V$ such that $\delta\left(s_{0}, \alpha\right)=s$. We further denote $V X$ the set obtained by concatenating each sequence $\alpha \in V$ with each input $x \in X$. The set $V X$ is usually called a transition cover set since each transition of the FSM $A$ is traversed with an appropriate sequence of the set $V X$.

Given state $s \in S$ of the FSM $A$, a finite set $W_{s}$ of finite input sequences is called $a$ state identifier of the state $s$ if for each state $p \in S, p \neq s$, there exists an input sequence $\alpha \in W_{s}$ such that $\lambda(p, \alpha) \neq \lambda(s, \alpha)$. An input sequence $\alpha$ is said to be a distinguishing sequence of the FSM $A$ if the output responses of the FSM $A$ at different states to $\alpha$ are different. When the FSM $A$ has a distinguishing sequence $\alpha$ then the set $\{\alpha\}$ is a state identifier of any state of $A$. When a state identifier $W_{s}$ is fixed for each state $s \in S$ a procedure for derivation of a complete test suite w.r.t. the fault model $\angle A, \cong$, $\Re_{n}>$ comprises two phases.

In the former phase, the part $T_{1}$ of the test suite is derived. The $T_{1}$ checks whether an implementation FSM has exactly $n$ states and each state identifier preserves its feature. This part of a test suite is obtained by concatenating each sequence $\alpha \in V$ with each sequence of the union of state identifiers $W_{s}$ over all states $s \in S$. If the implementation and reference FSMs have one and the same output sequence to each sequence of $T_{1}$ then any state of the reference FSM has a corresponding state in the implementation FSM with the same state identifier.

The part $T_{2}$ of a test suite derived in the latter phase, checks whether every transition of the implementation FSM is correctly implemented. The $T_{2}$ is obtained by concatenating each sequence $\alpha \in V X$ with each sequence of the state

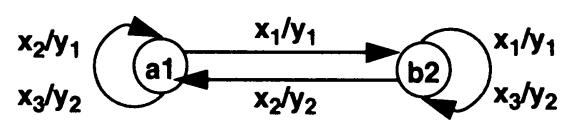

Figure 1 FSM $R S$. identifier of the state $\delta(s, \alpha)$ where $\alpha$ takes the FSM $A$ from the initial state. Merging the $T_{1}$ and $T_{2}$ we obtain a complete test suite $T S=T_{1} \cup T_{2}$ w.r.t. the fault model $\angle A$, $\cong \Re_{n}>$. For the sake of simplicity, each sequence that is a prefix of another sequence can be deleted from the test suite $T S$.

Example 1. Consider an FSM $R S$ in Figure 1 ( $a l$ is the initial state). An input sequence $x_{2}$ is a distinguishing sequence of the $R S$. We select the state cover set $V=$ $\left\{\varepsilon, x_{1}\right\}$. Then $T_{1}=\left\{x_{2}, x_{1} x_{2}\right\}$ while the transition cover set $V X=\left\{x_{1}, x_{2}, x_{3}, x_{1} x_{1}\right.$, $\left.x_{1} x_{2}, x_{1} x_{3}\right\}$. Concatenating each sequence of $V X$ with $x_{2}$ we obtain $T_{2}=\left\{x_{1} x_{2}, x_{2} x_{2}\right.$, $\left.x_{3} x_{2}, x_{1} x_{1} x_{2}, x_{1} x_{2} x_{2}, x_{1} x_{3} x_{2}\right\}$. After deleting from $T_{1} \cup T_{2}$ all prefixes of other sequences the set $T S=\left\{x_{2} x_{2}, x_{3} x_{2}, x_{1} x_{1} x_{2}, x_{1} x_{2} x_{2}, x_{1} x_{3} x_{2}\right\}$ is obtained that is a complete test suite w.r.t. the fault model $\angle R S, \cong, \Re_{2}>$. The tree of the test suite is shown in Figure 2. The test suite comprises five test cases of total length 18. 


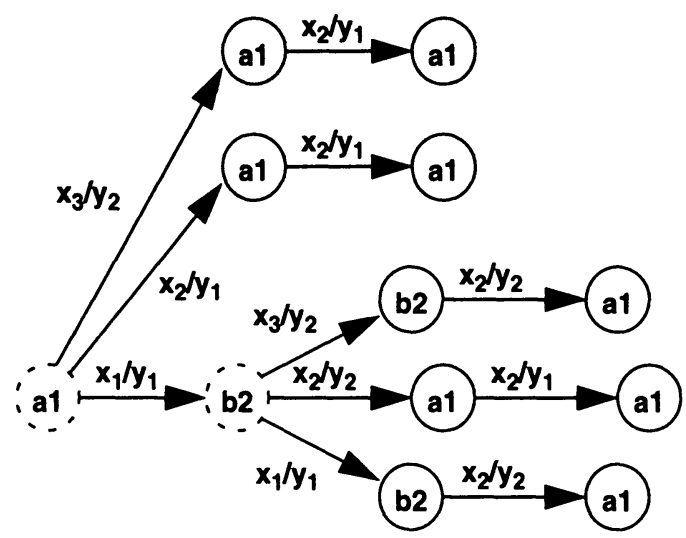

Figure 2 The tree of a complete test suite w.r.t. the fault model $\angle R S, \cong, \Re_{2}>$. States that should be identified are depicted in dashed lines.

\subsection{Composition of FSMs}

We consider a composition as a system of two component FSMs, as shown in Figure 3. For the sake of simplicity, we consider pairwise disjoint sets $X, Y, U$ and $\mathrm{Z}$. The system at hand has a single message in transit, i.e. the environment submits the next external input to the system only when it has produced an external output to the previous input. This accounts for synchronous communication between the environment and the system. Notice, however, that asyn-

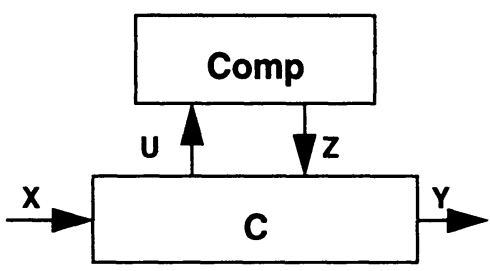

Figure 3 Composition of two FSMs: the context $C$ and the component Comp.

chronous communication through bounded input queues can be simulated by explicitly introducing new contexts representing the behaviour of the bounded queue in the system [Tretmans 92][Phalippou 94]. The input queues must be bounded, so to assure that the corresponding FSM exists (i.e. it is really finite).

Under these assumptions, when there are no live-locks in the composition, we can derive the composite machine of FSMs $C$ and Comp using, for instance, the algorithm described in [Lima 97] in order to avoid generating the Cartesian product CxComp (which is often huge).

Example 2. Consider the two machines $C$ and Comp shown in Figure $4 \mathrm{a}$ and Figure 4b. Considering states $a$ and $l$ the initial states, the composite machine $R S=$ C. Comp is the FSM of Figure 1.

We now demonstrate that the composition of Figure 3 is general enough to discuss the problems related to testing a component machine. We claim that the gen- 


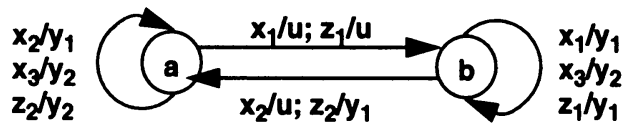

(a)

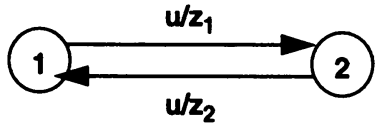

(b)

Figure 4 The context $C(\mathrm{a})$ and the component $\operatorname{Comp}(\mathrm{b})$.

eral model of Figure 5 can be transformed into that in Figure 3 with the component and composite FSM being isomorphic to those of the original composition.

If the external actions $X^{\prime}$ and $Y^{\prime}$ are redirected through the context along with the replacement of the external alphabets $X^{\prime}$ and $Y^{\prime}$ by the internal alphabets $U^{\prime}$ and $Z^{\prime}$ (Figure 6 ), we obtain an equivalent (isomorphic) system due to one-to-one correspondence. For each input $x \in X^{\prime}$, the context $C$ at any state produces a corresponding internal output $u \in U^{\prime}$ while for each internal input $z \in Z^{\prime}$ the context produces the corresponding $y \in Y^{\prime}$. Comp is isomorphic to EComp w.r.t. the oneto-one correspondence between $X^{\prime}$ and $U^{\prime}$

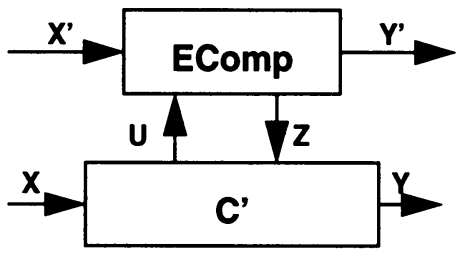

Figure 5 General composition of two FSMs. The context $C^{\prime}$ and the component EComp have external inputs and outputs. and $Y^{\prime}$ and $Z^{\prime}$ while the composite FSMs of the compositions coincide.

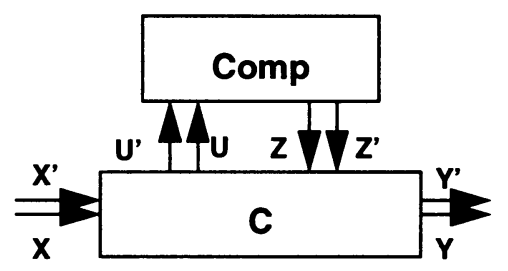

Figure 6 The transformed composition of two FSMs, the context $C$ and the component Comp.

\subsection{Fault model for testing in context}

Many compound systems are formally specified as a system of interacting FSMs. If only some components of a system need testing we face the problem of testing an embedded component or testing in context. In this case, a system under test can be represented as the composition of two FSMs. As stated in Section 1, one of these machines, called the component, is the composite FSM of all component machines which need testing while another machine, called the context, is the composite machine of all other component machines that are assumed to be faulty-free. As demonstrated above, we can use the composition in Figure 3 to discuss problems related to testing in context. 
We further suppose that the behaviour of a specification system is described by a deterministic FSM represented in the tester by a test suite. The tester executes test cases against an implementation system usually called a System Under Test (SUT), and checks the results on receipt of a response from the SUT. In this paper, we suppose that the SUT's behaviour can be represented by a deterministic composite machine. If the output response of the SUT to a proper test case differs from that of the specification system, then the tester produces the verdict 'fail'; in this case, the SUT is a nonconforming implementation of the specification system. Otherwise, the tester produces the verdict 'pass' to the test case. If the verdict 'pass' is produced to every test case of the test suite then we conclude that the SUT is a conforming implementation of the specification system.

Let a specification system be given as the composition of two interacting FSMs: the context $C$ and the component Comp. Let this composition be represented by the composite machine $R S$ with $n$ states. An implementation system is also the composition of two interacting machines: the context $C$ (a faultless implementation of the specification context $C$ ) and an implementation component machine Imp. Let the implementation system's behaviour be represented by the composite machine $I M$ with at most $n$ states. If $\Re_{n, C}$ is the set of all composite machines of such implementation systems, then we are required to derive a complete test suite w.r.t. the fault model $<R S, \cong, \Re_{n, C}>$, i.e. a set of input sequences of $R S$ such that for each implementation system of the domain that exhibits a different external behaviour from that of $R S$, there exists at least one test case when the tester produces the verdict 'fail'.

As mentioned above, regular methods exist for the derivation of a complete test suite w.r.t. the fault model $<R S, \cong, \Re_{n}>$. The test suite is also complete w.r.t. $<R S, \cong$, $\Re_{n, C}>$ because $\Re_{n, C} \subseteq \Re_{n}$. The test suite checks the component as well, but it also checks (unnecessarily) the context that is assumed to be faulty-free; thus, it can be reduced in particular cases. In the paper [Lima 97], some sufficient conditions for reducing the test suite are established. These conditions are briefly summarized in the following section (Section 3.1).

\section{DEALING WITH REDUNDANT TRANSITIONS}

\subsection{Reducing a test suite}

Each transition of the composite machine $R S$ comes from a combination of transitions of the two modules, the context and the component, but there may be transitions in the composite machine which are not affected by the component. In paper [Lima 97], such transitions are called redundant transitions; otherwise, a transition of the $R S$ is called suspicious. If the context is assumed to be faulty-free then external sequences traversing only redundant transitions are superfluous and can be deleted from the test suite without loss of its completeness. A method for determining such transitions is proposed and sufficient conditions for deleting superfluous test cases are established. These conditions can be formalized as follows. 
Proposition 3.1. Given the reference composite machine $R S$, let $\beta$ be an external input sequence only traversing redundant transitions of the $R S$. The output sequence of an implementation system to $\beta$ coincides with that of FSM $R S$.

Corollary 3.2. Given a complete test suite $T S$ w.r.t. the fault model $<R S$, $\cong$, $\Re_{n, C}>$, let $\beta \in T S$ be a sequence only traversing redundant transitions of the $R S$. Then $T S \backslash\{\beta\}$ is also a complete test suite w.r.t. the fault model.

Example 3. Consider sequences $x_{2} x_{2}$ and $x_{3} x_{2}$ of the complete test suite $T S$ w.r.t. the fault model $<R S, \cong, \Re_{2}>$ obtained in Section 2.2 . The sequences only traverse redundant transitions $\left(a 1, x_{2}\right),\left(a 1, x_{2}\right)$ and $\left(a 1, x_{3}\right),\left(a 1, x_{2}\right)$ of the composite FSM $R S$. Thus, $T S=\left\{x_{1} x_{1} x_{2}, x_{1} x_{2} x_{2}, x_{1} x_{3} x_{2}\right\}$ is a complete test suite w.r.t. the fault model $<R S, \cong, \Re_{2, C}>$. It has three sequences of total length 12 . All sequences of the set $T S$ traverse a suspicious transition.

Nevertheless, it was pointed out that a more rigorous analysis is necessary in order to elaborate a method for checking suspicious transitions.

\subsection{Redundant transitions}

It may well happen that a redundant transition of the specification system becomes suspicious due to an unexpected interaction in the implementation system that brings the context to a wrong state. Consider, for instance, the system of two FSMs: context $D$ (Figure 7a) and component Spec (Figure 7b).

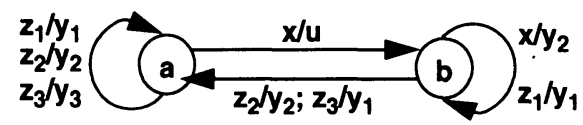

(a)

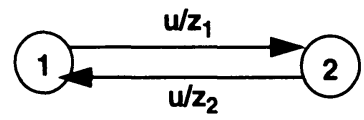

(b)

Figure 7 The context $D(\mathrm{a})$ and the component $\operatorname{Spec}(\mathrm{b})$.

The composite FSM is shown in Figure 8.

We use input sequence $x$ to reach state $b 2$ from the initial state $a l$ and the same sequence $x$ as a distinguish-

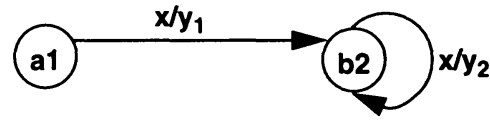

Figure 8 The composite FSM D.Spec. ing sequence. Thus, $T_{1}=\{x, x x\}$ coincides with $V X=\{x, x x\}$ and $T_{2}=\{x x, x x x\}$, i.e. $T S=\{x x x\}$ is a complete test suite w.r.t. the fault model $\left\langle D \cdot\right.$ Spec, $\left.\cong, \Re_{2}\right\rangle$. Note that the transition from the state $b 2$ under input $x$ is a redundant transition. If we do not check this transition, then $T S^{\prime}=$ $\{x x\}$. However, $T S^{\prime}$ is not complete w.r.t. the fault model $\left\langle D \cdot S p e c, \cong, \Re_{2, C}\right\rangle$.

Assume that the implementation component FSM Imp depicted in Figure 9a replaces Spec. The composite FSM $D \cdot I m p$ is shown in Figure $9 \mathrm{~b}$. By direct inspection, one can assure that the input sequence $x x x$ is the shortest sequence distinguishing nonequivalent FSMs $D \cdot$ Spec and $D \cdot I m p$.

The reason is that an implementation component machine, being an arbitrary FSM over alphabets $U$ and $Z$, can induce unexpected internal interactions in the implementation system. These unexpected internal interactions bring the implemen- 


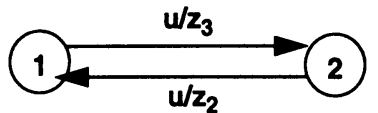

(a)

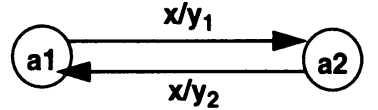

(b)

Figure 9 The implementation component FSM $\operatorname{Imp}$ (a) and the implementation composite FSM $D \cdot \operatorname{Imp}(\mathrm{b})$.

tation system to a wrong state while producing the expected output. These kinds of faults are known as latent faults [Petrenko 96-2] and can only be detected when longer external test cases are submitted. Therefore, some effort must be put into characterizing such cases, so to improve the proposed solution.

Given an external input sequence $\alpha \in X^{*}$ of the FSM $R S$ and an internal trace over alphabets $U$ and $Z$, the trace is said to be unexpected w.r.t. $\alpha$ if the trace may be induced in an implementation system not coinciding with that of the specification system when $\alpha$ is submitted. Unexpected internal traces represent alternative paths that a system may take due to faults in the implementation component machine.

Using the concept of unexpected internal traces in the previous example, we observe that a latent fault appears because:

1. there exist unexpected internal traces $u z_{2}$ and $u z_{3}$ w.r.t. the external input $x$; and

2. one of these unexpected internal traces, namely $u z_{3}$, produces the expected external output $y_{1}$.

Generally speaking, given a redundant transition of the FSM $R S$ at state $s$, the latent fault may occur under the following conditions:

1. the external input sequence $\alpha \in V X$ that takes the $R S$ from its initial state to state $s$ traverses suspicious transitions; and

2. at least one of the unexpected internal traces w.r.t. $\alpha$ results in the expected output sequence.

Based on this observation we conclude that a redundant transition becomes suspicious if conditions 1 and 2 above apply, and then the tail state of such transition must be checked as well.

On the other hand, if for some test case of a given test suite, none of the unexpected internal traces w.r.t. the case result is the expected external output sequence, then the output sequence of an implementation to the test case coincides with that of the specification system. In this case, the test case is superfluous and can be deleted from the test suite without loss of its completeness. When at least one of the unexpected internal cases w.r.t. the test case results in an unexpected external output sequence, other test case should be examined to recognize those that are superfluous. 


\section{TEST SUITE MINIMIZATION}

\subsection{Detectable internal traces}

The example of Section 3.2 clearly demonstrates that the fault detection power of an external input sequence $\alpha$ in the given context can be characterized by the set of all unexpected internal traces such that, if an implementation component machine comprises such a trace, then the specification and implementation systems produce different output sequences to $\alpha$. We call such internal traces detectable with the external input sequence $\alpha$. If an implementation component FSM has no traces detectable with $\alpha$, then the specification and implementation systems produce the same output sequence to $\alpha$. The formal definition of such internal traces is given in [Petrenko 97] where they are called forbidden internal traces w.r.t. $\alpha$. As the set of traces of an FSM is a prefix-closed set, an extension of a trace which is detectable with an external input sequence is also detectable with this sequence. Conversely, a set of internal traces that are detectable with an external input sequence includes that for its prefix.

Given a set $T$ of external input sequences, an internal trace is said to be detectable with the set $T$ if it is detectable with at least one sequence of the set. Due to the definition of detectable traces, the following statement holds.

Proposition 4.1. Given the composite FSM $R S$ of the specification system with the context $C$ and a complete test suite $T S$ w.r.t. fault model $\left\langle R S, \cong, \Re_{n, C}\right\rangle$, let $P$ be a subset of $T S$. If all internal traces detectable with $T S$ are detectable with $P$ then $P$ also is a complete test suite w.r.t. the fault model.

Due to Proposition 4.1, to compare the fault detection power of two test suites we need a procedure for characterizing internal traces that are detectable with a given external input sequence. We represent such traces as sequences recognized by a designated final state 'fail' of an appropriate acceptor or recognizer [Hopkroft 79]. Our first step is to describe all possible traces that may be induced in an implementation system when the external sequence is submitted. Because a component FSM is completely embedded within the context (see Section 2.3), these traces can be described as traces of the context LTS $L C$ obtained by unfolding each atomic transition input/output in the context FSM $C$. The context LTS $L C$ for the context $C$ in Figure 4a is shown in Figure 10.

Given an external input $x \in X$, we construct the acceptor $L C(x)$. States of the acceptor are states of $L C$ with the initial state $s_{0}$. The initial and final states of the acceptor are special states that cannot be merged with other states with the same names called intermediate states, while it is possible to merge two such intermediate states. There is a transition labelled with $x$ from the initial state to state $s$ if $x$ takes the $L C$ from the initial state $s_{0}$ to the state $s$. For two intermediate states $p$ and $r$, there is a transition labelled with action $a \in U \cup Z$ if there is a transition labelled with $a$ from state $p$ to state $r$ in the LTS $L C$. There is a transition labelled with $y \in Y$ from the intermediate state $p$ to the final state $s$ if there exists an outgoing transition 
at the state $p$ to state $s$ labelled with $y$ in the LTS $L C$. The connected part of the acceptor comprising the initial state is denoted $L C(x)$.

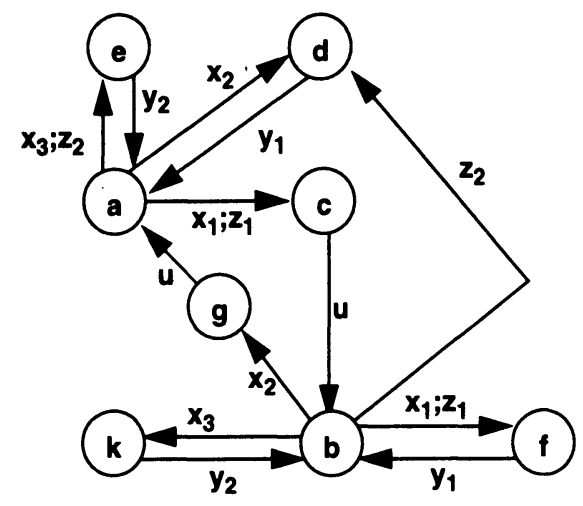

Figure 10 The context LTS $L C$ representing all the traces of the context $C$.

Given a final state $s$ of the acceptor $L C(x)$, the set of all sequences labelling the paths from the initial to the final state $s$ is a regular set recognized by the final state $s$ [Hopkroft 79]. Due to construction of the acceptor, the regular expression specifying the set can be written as $x f y$ where $f$ comprises only internal actions of the set $U \cup Z$. Thus, the set of the $(U \cup Z)$-projections of the regular set recognized by a final state also is a regular set specified by a proper regular expression.

To construct the acceptor $L C\left(x_{1} \ldots x_{k}\right)$ we construct at each terminal state $s$ of the acceptor $L C\left(x_{l}\right)$ the acceptor $L C\left(x_{2}\right)$ with $s$ as the initial state. The construction of the acceptor $L C\left(x_{1} \ldots x_{k}\right)$ implies the following statement.

Proposition 4.2. Given the acceptor $L C\left(x_{1} \ldots x_{k}\right)$, let $I S$ be the implementation system of a component over alphabets $U$ and $Z$ within context $C$. 1) If $\sigma$ is a trace of the system when the external input sequence $x_{l} \ldots x_{k}$ is submitted, then the acceptor $L C\left(x_{1} \ldots x_{k}\right)$ has a path from the initial state to a final state labelled with the sequence $\sigma$. 2) If an $(U \cup Z)$-projection of sequence $\sigma$ labelled a path from the initial to a final state is a trace of the implementation component, then the output sequence of the implementation system to $x_{1} \ldots x_{k}$ is the $Y$-projection of the sequence $\sigma$.

Example 4. Consider an implementation system with the context of Figure 4a and an input sequence $x_{1} x_{2} x_{2}$. When the external input $x_{1}$ is applied to the context at the initial state $a$ the context produces the internal output $u$ and enters the state $b$. Its next action depends on an output produced by a component machine to the input $u$. If the component produces $z_{1}$ then the context remains at the state $b$ producing $y_{1}$ while the context enters the state $a$ producing $y_{1}$ if the component has the output response $z_{2}$ to $u$. In both cases, the context produces an external output $y_{1}$ to $x_{1}$ and the next external input $x_{2}$ can be applied to the context. Since we are interested in all the traces that may be induced when the external input sequence is submitted to the context we do not merge states with the same names separated with external actions. The procedure of constructing the acceptor $L C\left(x_{1} x_{2} x_{2}\right)$ is illustrated in Figure 11. $\square$

The next step is to transform the acceptor, so that the set of all internal traces detectable with the given external input sequence would be represented by the set of $(U \cup Z)$-projections of the sequences recognized by a designated state 'fail.'

Procedure 4.1. Derivation of the regular expression for forbidden traces w.r.t. a given external input sequence. 
Inputs: The composite machine $R S$ of the specification system with a context $C$ and an external input sequence $\alpha$.

Output: A regular expression $E_{\alpha}$ for internal traces detectable with $\alpha$.

Step 1. Derive the context LTS $L C$ unfolding each atomic transition input/ output in the context FSM $C$ and construct the acceptor $L C(\alpha)$.

Step 2. For each path of the acceptor $L C(\alpha)$ from the initial state to a final state such that the $(X \cup Y)$ - projection of the sequence labelled the path is not a trace of the specification composite FSM $R S$, replace the last state of the path with a designated deadlock state 'fail.'

Step 3. If for some transition labelled with external input $x \in X$ or with an internal action $z \in Z$, all the subsequent paths have a final state 'fail' then replace the final state of the transition with the 'fail' state.

Step 4. Derive a regular expression $E_{\alpha}$ for $(U \cup Z)$ - projections of sequences labelling all paths from the initial state to FAIL state.

The regular language obtained by Procedure 4.1 , is called the characterization fault detection set $D(\alpha)$ of the sequence $\alpha$ which completely characterizes the set of nonconforming implementation systems that can be detected with the external input sequence $\alpha$.

Proposition 4.3. Given a context $C$, a component specification Comp, a component implementation Imp the composite

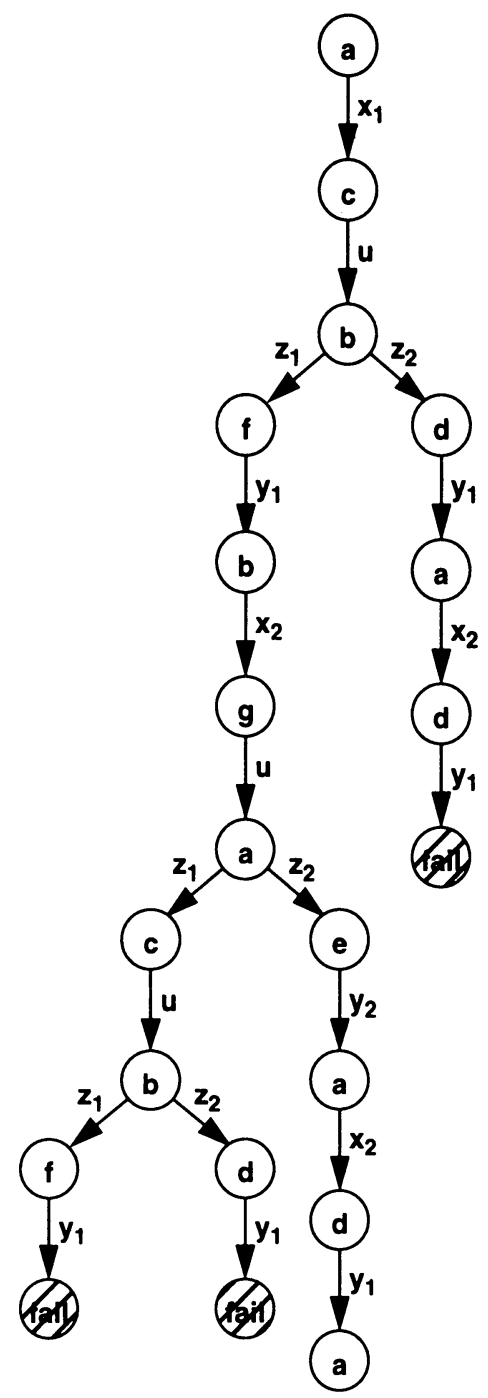

Figure 11 The fragment of the acceptor $L C\left(x_{1} x_{2} x_{2}\right)$. The external projections of executed traces are underlined. machines $R S$ (system specification) and $I S$ (system implementation), and an external input sequence $\alpha$, let $E_{\alpha}$ be a regular expression obtained by the Procedure 4.1. The external sequence $\alpha$ distinguishes FSMs $R S$ from $I S$ iff the language specified by the expression $E_{\alpha}$ comprises a trace of the component Imp of the implementation system.

Proof. First part. Let the language specified by the regular expression $E$ comprise a trace $\beta / \gamma$ of a component Imp. By construction of $E_{\alpha}$, there is a path in the 
acceptor (obtained at Step 2 of Procedure 4.1) from the initial state to the 'fail' state labelled with a sequence such that: 1) its $(U \cup Z)$ - projection $\beta \beta^{\prime} / \gamma \gamma^{\prime}$ is a trace of the implementation component $\operatorname{Imp} ; 2$ ) its $X$-projection is $\alpha$; and 3) its $Y$-projection $\sigma$ is different from the $R S$ 's output sequence to $\alpha$. The 'fail' state replaces an appropriate final state of the acceptor $L C(\alpha)$. Due to Proposition 4.2 (Part 2), the implementation system produces the output sequence $\sigma$ when $\alpha$ is submitted to the system.

Second part. We now assume that the $I S$ has the output sequence $\sigma$ to $\alpha$ that is different from that of the $R S$. Then the acceptor obtained at Step 2 of Procedure 4.1 comprises the path from the initial state to the 'fail' state labelled with a trace induced in the implementation system when $\alpha$ is submitted to the system (Proposition 4.2, Part 1). Therefore, the $X$ - projection of the sequence labelling the path is $\alpha$, the $Y$ - projection is $\sigma$ and the $(U \cup Z)$ - projection is a trace $\beta / \gamma$ of the component Imp. By construction, the language specified by regular expression $E$ comprises a prefix to $\beta /$ $\gamma$, i.e. a trace of the component Imp.

Corollary 4.4. Given an internal trace $\tau$ and an external input sequence $\alpha, \tau$ is detectable with $\alpha$ iff the set $D(\alpha)$ contains a prefix of $\tau$.

Given the set $T$ of external input sequences, the union of the characterization fault detection sets over all sequences in the set $T$ is called the characterization fault detection set $D(T)$ of $T$.

Example 5. Consider a sequence $x_{1} x_{2} x_{2}$ of the complete test suite $T S=\left\{x_{1} x_{1} x_{2}\right.$, $\left.x_{1} x_{2} x_{2}, x_{1} x_{3} x_{2}\right\}$ w.r.t. the fault model $\left\langle R S, \cong, \Re_{2, C}\right\rangle$ obtained in Section 3 after deleting test cases traversing only redundant transitions. This sequence traverses suspicious transitions. We need now to determine the characterization fault detection set $D\left(x_{1} x_{2}\right)$ for its prefix $x_{1} x_{2}$.

Consider in the acceptor the path labelled with the sequence $x_{1} u z_{2} y_{1} x_{2} y_{1}$ from the initial state. A system executes this sequence of actions when the context is combined with an implementation component machine producing at the initial state the output $z_{2}$ to the input $u$. Once the component of a system produces the output $z_{2}$ to the input $u$, the system will produce the output sequence $y_{1} y_{1}$ to the external input sequence $x_{1} x_{2}$. This output does not coincide with the expected output $y_{1} y_{2}$ of the reference composite FSM (Figure 1) to the input $x_{1} x_{2}$. Thus, the final state of the path is replaced by a 'fail' state.

Consider now the paths labelled with sequences $x_{1} u z_{1} y_{1} x_{2} u z_{1} u z_{1} y_{1}$ and $x_{1} u z_{1} y_{1} x_{2} u z_{l} u z_{2} y_{1}$. A system executes these sequences of actions when the context is combined with an implementation component machine producing at the initial state the output sequence $z_{1} z_{1} z_{1}$ or $z_{1} z_{1} z_{2}$ to the input sequence $u u u$. Thus, since the implementation component of a system at hand produces the output sequence $z_{1} z_{1} z_{1}$ (or $z_{l} z_{l} z_{2}$ ) to the input sequence $u u u$, the system produces an output sequence $\left(y_{l} y_{l}\right)$ to the input sequence $x_{1} x_{2}$ that is different from the expected output sequence $y_{1} y_{2}$ of the reference FSM $R S$ (Figure 1). The prefix $x_{1} u z_{1} y_{1} x_{2} u z_{1}$ of the sequences takes the acceptor from the initial state to a state where all the subsequent paths lead to a 'fail' state. In other words, if an implementation component machine has a trace $u z_{l} u z_{l}$, then the system will always produce the unexpected output sequence $y_{l} y_{l}$ to the input sequence $x_{1} x_{2}$, regardless of the output of the implementation component 
to the next input $u$. That is why we replace the state where $x_{1} u z_{1} y_{1} x_{2} u z_{1}$ takes the acceptor to from the initial state by a 'fail' state. Therefore, $D\left(x_{1} x_{2}\right)=\left\{u z_{1} u z_{1}, u z_{2}\right\}$. By direct inspection of Figure 11, one can assure the set $D\left(x_{1} x_{2} x_{2}\right)$ coincides with $D\left(x_{1} x_{2}\right)$, i.e. the test case $x_{1} x_{2} x_{2}$ can be replaced by $x_{1} x_{2}$ without loss of completeness of the TS w.r.t. the fault model $\left\langle R S, \cong, \Re_{2, C}>\right.$.

On the other hand, if the component of an implementation system has no traces of the set $D\left(x_{1} x_{2}\right)$, then even unexpected internal interactions in an implementation system result in the expected external output sequence $y_{1} y_{2}$ of the reference FSM $R S$ to the external sequence $x_{1} x_{2}$. In a similar way, we determine the sets $D\left(x_{1} x_{1} x_{2}\right)$ $=\left\{u z_{2} u z_{2}, u z_{2} u z_{1} u z_{1}, u z_{1} u z_{1}\right\}$ and $D\left(x_{1} x_{3} x_{2}\right)=\left\{u z_{2}, u z_{1} u z_{1}\right\}$. Thus, the fault characterization set $D(T S)=\left\{u z_{2} u z_{2}, u z_{2} u z_{1} u z_{1}, u z_{2}, u z_{1} u z_{1}\right\}$ comprises a prefix of any internal trace detectable with the test suite $T S$. $\square$

Once the component of an implementation system has a trace belonging to $D(T S)$, there exists a test case in the $T S$ to which the implementation and reference systems produce different output sequences. Conversely, if the component of an implementation system has no traces of the set $D(T S)$ then the implementation and reference systems produce the same output sequence to each test case of the TS. In other words, if the composite machine of the implementation system has at most two states then it is equivalent to the $R S$. That is to say that all components having no traces of the set $D(T S)$ that combined with the context possess the composite machine with at most two states are equivalent to the specification component Comp in the context $C$. At the PCOs it is impossible to recognize which of them serves as the component of the system at hand.

\subsection{Minimizing a test suite}

\section{Removing redundant transitions}

Using the results of Section 3 and Section 4.1, we can reduce the procedure of derivation of a complete test suite w.r.t. the fault model $<\mathrm{A}, \cong, \Re_{n}>$ (as explained in Section 2.2) to the procedure to generate a complete test suite w.r.t. the fault model $<\mathrm{A}, \cong, \Re_{n, C}>$.

Procedure 4.2. Deriving a complete test suite w.r.t. the fault model $<\mathrm{A}$, $\cong$, $\Re_{n, C}>$.

Input: The composite FSM $R S$ of a specification system with $n$ states that is minimal and connected; the state cover set of $R S$; a transition cover set; and the set of state identifiers for all the states of $R S$.

Output: A complete test suite w.r.t. the fault model $<\mathrm{A}, \cong, \Re_{n, C}>$.

Step 1. Concatenate each sequence of the state cover set with each sequence of the union of the state identifiers over all states of the FSM $R S$. Denote $T_{1}$ the set obtained. Let $Q$ ' be the subset of states of the context such that, for any state $q \in Q^{\prime}$, there exists a sequence of the state cover set traversing only redundant transitions taking the context from the initial state to state $q$.

Step 2. Concatenate each sequence of the transition cover set traversing either a 
suspicious transition or taking the FSM $R S$ from the initial state to state $(q, t)$, $\left(q \notin Q^{\prime}\right)$ with each sequence of the identifier for the state to which the sequence takes the FSM $R S$ from the initial state. Denote $T_{2}$ the set obtained and $T S$ the union of $T_{1}$ and $T_{2}$. Each sequence that is a prefix of another sequence can be deleted from the $T S$.

Comparing the above procedure with that in Section 2.2 we do not include in $T_{2}$ any subset obtained by concatenating a sequence of the transition cover set traversing only redundant transitions and taking the FSM $R S$ from the initial state to the state $(q, t)$, where $q \in Q^{\prime}$, with an appropriate state identifier, i.e. we do not test an unnecessary transition [Lima 97].

Proposition 4.5. Given the composite FSM $R S$ of the specification system with $n$ states, the set $T S$ derived by Procedure 4.2 is a complete test suite w.r.t. the fault model $\left\langle R S, \cong, \Re_{n, C}\right\rangle$.

Proof. Let $\alpha$ be a sequence of the state cover set traversing only redundant transitions and taking the FSM $R S$ from the initial state to state $(q, t)$, where $q \in Q^{\prime}$. Moreover, let $\beta$ be a sequence of the transition cover set traversing only redundant transitions and taking the FSM $R S$ from the initial state to the state $\left(q, t^{\prime}\right)$. By construction, the set $T_{l}$ contains all sequences of the state identifier $W_{q t^{\prime}}$ for state $\left(q, t^{\prime}\right)$. Each internal trace detectable with the sequence $\beta \gamma, \gamma \in \mathrm{W}_{\mathrm{qt}}$, is detectable with $\alpha \gamma$ because the parts of both acceptors $L C(\beta \gamma)$ and $L C(\alpha \gamma)$ comprising internal traces start at the same initial state $q$ of the context. Thus, the subset containing the concatenation of the subsequence $\beta$ with each sequence of the set is superfluous in the complete test suite w.r.t. the fault model $\left\langle R S, \cong, \Re_{n, C}>\right.$.

\section{Deleting superfluous test cases}

Suppose now that we derive a complete test suite w.r.t. the fault model $<R S, \cong$, $\Re_{n, C}>$ using Procedure 4.2 (i.e. not including into the test suite a sequence traversing only redundant transitions - Corollary 3.2). There may exist a proper subset of the test suite such that any internal trace detectable with the test suite is detectable with the subset, i.e. some sequences of the test suite may still be superfluous. Due to Proposition 4.3, to compare a fault detection power of two external input sequences, it is sufficient to compare the corresponding regular sets (the comparison of arbitrary regular sets is out of the scope of this paper). Let us assume that the characterization fault detection set $D(T S)$ is finite for a given complete test suite $T S$ w.r.t. the fault model $\left\langle R S, \cong, \Re_{n, C}\right\rangle$, as it often occurs in practical situations. In this case, the problem of determining a minimal subset of a complete test suite $T S$, that is also complete w.r.t. $\left\langle R S, \cong, \Re_{n, C}>\right.$, is reduced to derivation of a minimal column coverage $P$ of the boolean matrix $B$, i.e. a minimal row subset of $P$ such that for each column there exists at least one row of $P$ comprising $1 \mathrm{~s}$ in this column. The rows of the matrix $B$ correspond to sequences of the test suite $T S$ while the columns correspond to internal traces of the characterization fault detection set $D(T S)$. Item $b_{i j}$ is ' 1 ' iff 
for the sequence $\alpha_{\mathrm{i}} \in T S$, the characterization fault detection set $D\left(\alpha_{\mathrm{i}}\right)$ contains a prefix of the internal trace $\left(\beta / \gamma_{\mathrm{j}} \in D(T S)\right.$.

Example 6. The boolean matrix $B$ for our working example is shown in Table 1. By direct inspection, one can assure that set $D\left(x_{1} x_{2}\right)$ contains the prefixes of all traces of the set $D(T S)$, i.e. all internal traces detectable with the test suite $T S=$ $\left\{x_{1} x_{1} x_{2}, x_{1} x_{2}, x_{1} x_{3} x_{2}\right\}$ (obtained in Section 4.1) are detectable with the external input sequence $x_{1} x_{2}$. Therefore, the subset $\left\{x_{1} x_{2}\right\}$ is a complete test suite w.r.t. the fault model $<R S, \cong, \Re_{2, \mathrm{C}}>$ being sufficient to detect an implementation system that possesses the composite machine with at most two states and is not equivalent to the reference composite FSM $R S$ in Figure 1 when the context is faulty-free. One can compare this test suite (of total length 2) with a complete test suite w.r.t. the fault model $<R S, \cong, \Re_{2}>$ obtained in Section 2.2 that comprises five test cases of total length 18 .

In a number of practical situations it is nearly impossible to derive the composite machine of the overall system due to its huge number of states. In this case, a test suite often is derived by a test engineer who is a high-level expert in the area. The obtained test suite checks important features of the protocol's implementations but it is difficult to estimate its fault coverage in the formal way. The above approach can be used to reduce the given test preserving its fault detection power.

Let a specification system be a composition of the context $C$ and the component Comp possessing the composite machine $R S$ and let $\Re$ be a finite set of FSMs over the same input alphabet as $R S$. We denote $\Re_{C}$ the subset of $\Re$ comprising each machine of the set $\Re$ that is the composite machine of some implementation system with the same context. Given a set $T$ of test cases that is complete w.r.t. the fault model $<R S, \cong, \Re_{C}>$, a subset $P$ of the set $T$ is said to have the same fault detection power in the given context if it is also a complete test suite w.r.t. that fault model.

TABLE 1. The boolean matrix B

\begin{tabular}{l|llll}
\hline & \multicolumn{5}{c}{$\mathbf{u}$} & & \\
& & $\mathbf{z}_{2}$ & & \\
& $\mathbf{u}$ & $\mathbf{u}$ & $\mathbf{u}$ & \\
& $\mathbf{z}_{2}$ & $\mathbf{z}_{1}$ & $\mathbf{z}_{1}$ & \\
& $\mathbf{u}$ & $\mathbf{u}$ & $\mathbf{u}$ & $\mathbf{u}$ \\
& $\mathbf{z}_{2}$ & $\mathbf{z}_{1}$ & $\mathbf{z}_{1}$ & $\mathbf{z}_{2}$ \\
\hline $\mathrm{x}_{1} \mathrm{x}_{1} \mathrm{x}_{2}$ & 1 & 1 & 1 & 0 \\
$\mathrm{x}_{1} \mathrm{x}_{2}$ & 1 & 1 & 1 & 1 \\
$\mathrm{x}_{1} \mathrm{x}_{3} \mathrm{x}_{2}$ & 1 & 0 & 1 & 0
\end{tabular}

Given the context $C$ and the set $T$ of external test cases, we can use Procedure 4.1 to determine the characterization fault detection $D(T)$ and derive its subset $P$ with the same fault detection power as a minimal coverage of an appropriate boolean matrix when $D(T)$ is finite. 


\section{FUTURE WORK AND CONCLUSION}

Our future work is basically related to the generalization of the proposed approach. It can be easily generalized to the case when some transitions of the specification context and component are undefined while their implementations are assumed to be completely specified. In undefined situations, the implementation can produce an error message, for example, or have a loop labelled with the NULL output. In this case, conformance testing for protocols can be formalized as FSMs quasi-equivalence problem where a complete FSM $B$ is said to be quasi-equivalent to (possibly partial) FSM $A$ if $A$ and $B$ exhibit the same behaviour under all input sequences where a behaviour of $A$ is specified. We can call methods for a complete test suite derivation w.r.t. the fault model $\left\langle A, \cong_{q}, \Re_{n}\right\rangle$ [Petrenko 96-1][Yannakakis 95] (where $\cong_{q}$ is a quasi-equivalence relation) and use the method proposed in this paper to minimize the obtained test suite.

We also would like to generalize this approach to a system of communicating nondeterministic FSMs. As the authors of the paper [Lima 97] notice, Proposition 3.1 and Corollary 3.2 hold in this case. But now it is insufficient to keep in a test suite external sequences detecting each trace detectable w.r.t. the test suite. The subset of remaining test cases should also check whether the set of output responses of an IUT to an external input sequence $\alpha$ contains each output sequence of the reference composite FSM to $\alpha$. The problem is closely related to the problem of equation solving [Merlin 83][Kim 72][Parrow 89][Watanabe 93], where we are required to derive the largest specification of the sub-module that combined with the given context is equivalent to the specification's composite FSM. To the best of our knowledge the problem is not solved to the general case; solutions only exist for particular cases.

In this paper we presented a test derivation strategy for testing in context. The system studied was composed of two communicating FSMs, the context and component, and the test architecture was generic, i.e. the context and the component may have external inputs and outputs. Using the given fault model for testing in context, conditions were provided to detect redundant transitions that did not need to be tested.

Furthermore, given an external input sequence, a regular set was derived such that a nonconforming implementation system could be detected with this input sequence if and only if its component comprised a trace of the set. Based on this approach a method was also presented to construct a complete test suite and to select a minimal subset of this test suite having the same fault detection power. On the other hand, the proposed method can be used to reduce a test suite given by human experts while preserving its fault detection power. 


\section{REFERENCES}

[Chow 78] T. S. Chow, Test software design modelled by finite state machines, IEEE Trans. SE-4, No. 3, pp. 178-187, 1978.

[Fujiwara 91] S. Fujiwara, G. v. Bochmann, F. Khendek, M. Amalou, A. Ghedamsi, Test selection based on finite state models, IEEE Trans., SE-17, No. 6, 1991, pp. 591-603.

[Hopkroft 79] J. E. Hopkroft, J. D. Ulman, Introduction to automata theory, languages and computation, 1979, Addison-Wesley, NY.

[Huang 96] S. Huang, D. Lee, M. Stauskas, Validation-based test sequence generation for networks of extended finite state machines, Proc. of the 1st Joint Intern. Conf. on Formal Description Techniques for Distributed Systems and Communication Protocols., and Protocol Specification, Testing, and Verification. 1996, pp. 403-418.

[IS-9646 91] Information Technology, Open Systems Interaction, Conformance Testing Methodology and Framework, International Standard IS-9646. ISO, 1991.

[Kim 72] J. Kim, M. Newborn, The simplification of sequential machines with input restrictions, IEEE Trans. on Computers, C-20, pp. 1440-1443, 1972.

[Kim 95] M. C. Kim, S. T. Chanson, S. Yoo, Design for testability of protocols based on formal specifications, Proc. of the 8th Intern Workshop on Protocol Test Systems, 1995.

[Lee 96] D. Lee, K. K. Sabnani, D. M. Kristol, S. Paul, Conformance testing of protocols specified as communicating finite state machines - a guided random walk based approach, IEEE Trans. on Communication, 1996, vol. 44, 5, pp. 631-640.

[Lima 97] L. P. Lima, A. R. Cavalli, A pragmatic approach to generating test sequences for embedded systems, Proc. of the 10th Intern. Workshop on Protocol Test Systems, 1997.

[Lima 98] L. P. Lima, A. R. Cavalli, Application of embedded testing methods to service validation, submitted to 2nd IEEE International Conference on Formal Engineering Methods, Brisbane, Australia, 1998.

[Merlin 83] P. Merlin et al. On the construction of submodule specifications and communication protocols, ACM Trans. on Programming Languages and Systems. Vol. 5, No. 1, 83.

[Parrow 89] J. Parrow, Submodule construction as equation solving in CCS, Theoretical Computer Science, 68, 1989, pp. 175-202.

[Petrenko 97] A. Petrenko, N. Yevtushenko, Testing faults in embedded components, Proc. of the 10th Intern. Workshop on Protocol Test Systems, 1997.

[Petrenko 96-1] A. Petrenko, N. Yevtushenko, G. v. Bochmann, Testing deterministic implementations from nondeterministic FSM specifications, Proc. of the 9th Intern Workshop on Protocol Test Systems, 1996.

[Petrenko 96-2] A. Petrenko, N. Yevtushenko, G. v. Bochmann, Fault models for testing in context, Proc. of the 1st Joint Intern. Conf. on Formal Description Techniques for Distributed Systems and Communication Protocols and Protocol Specification, Testing, and Verification. 1996, pp. 125-140.

[Petrenko 92] A. Petrenko, N. Yevtushenko, Test suite generation for a given type of implementation errors, Proc. of the Intern. Conf. on Protocol Specification, Testing, and Verification. 1992.

[Phalippou 94] M. Phalippou, "Relations d'implantation et hypothèses de test sur des automates à entrées et sorties”, Ph.D. Thesis, Université de Bordeaux I, 1994.

[PT 96] Component testing for mobile and broadband telecommunications, COIMBRA, COPERNICUS project Proposal, 1996.

[Tretmans 92] J. Tretmans, “A Formal Approach to Conformance Testing”, Ph.D. Thesis, 1992. 
[Vasilevsky 73] M. P. Vasilevsky, Failure diagnosis of automata. Cybernetics, Plenum Publishing Corporation, NY, No. 4, 1973, pp. 653-665.

[Vuong 89] S. T. Vuong, W. W. L. Chan, M. R. Ito, The UIOv-method for protocol test sequence generation, Proc. of IFIP TC6 Second Inter. Workshop on Protocol test Systems. 1989, pp. 161-175.

[Watanabe 93] Y. Watanabe, R. K. Brayton, The maximal set of permissible behaviours for FSM networks. Proc. of the IEEE/ACM Intern. Conf. on Computer-Aided Design, 1993, pp. 316-320.

[Yannakakis 95] M. Yannakakis, D. Lee, Testing finite state machines: fault detection, Journal of Computer and System Sciences, 1995, 50, pp. 209-227.

\section{BIOGRAPHY}

Nina Yevtushenko received the Diploma degree in radio-physics in 1971 and Ph.D. in computer science in 1983, both from the Tomsk State University, Russia. She is currently a Professor at that University. Her research interests include the automata and FSM theory and testing problems.

Luiz Paula Lima Jr. is currently a PhD student at INT (Institut National des Télécommunications), Evry, France and he received his MSC degree in 1994 at UNICAMP (State University of Campinas), Brazil. His current research interests include object-oriented distributed systems and platforms (ODP/CORBA) and testing methods for these architectures.

Ana Rosa Cavalli received the Doctorat d'État es Mathematics and Computer Science in 1984 from the University of Paris VII, Paris, France. From 1985 to 1990, she was a staff research member at the CNET (Centre National d'Etudes des Télécommunications), where she worked on software engineering and formal description techniques. Since 1990, she joined the INT (Institut National des Télécommunications) as professor. Her research interests include formal description techniques, validation of protocols and services, computing methodology and testing methods for distributed architectures. 